\title{
THE MINNEAPOLIS COLLOQUIUM
}

The lectures by Professor Marston Morse were concerned with the new theory of the calculus of variations in the large developed by him within the last few years. The intimate relations existing between the calculus of variations and many other branches of modern mathematics were strikingly illustrated by these lectures. For example, combinatorial analysis situs and the theory of analytic functions of several variables form an essential part of the foundations for the new theory, which in turn adds to our knowledge about differential equations and boundary value problems, about dynamics, about "differential geometry in the large." Morse has also initiated a new chapter in analysis situs by studying the connectivity numbers of manifolds in function space associated with calculus of variations problems. Here there may occur infinite connectivity numbers, and infinitely many of the connectivity numbers may be non-zero.

The first lecture was introductory, giving a preliminary outline of methods and results. The nature of the results was indicated by means of simple examples. Then the theory associated with the type number or index of an extremal under general end conditions was developed. This theory includes separation, comparison, and oscillation theorems for systems of self-adjoint linear differential equations of the second order. The index of an extremal is defined by means of the characteristic numbers for an associated boundary value problem, and is shown to be equal to the negative type number of an associated quadratic form. The third lecture was devoted to the inequality relations between the numbers of different types of critical points of functions and the connectivity numbers of the manifolds on which they are defined. The fourth and final lecture took up the connectivities in function space associated with a calculus of variations problem and the inequality relations existing between the numbers of extremals of different types and the connectivities. These inequalities yield some extraordinary existence theorems on the number of extremals satisfying general end conditions. A number of interesting examples illustrated the results. 
[November,

The lectures were extremely stimulating and instructive. It is greatly to be hoped that the details of the theory outlined in them will soon appear in book form.

There follows the preliminary outline of the lectures prepared by Professor Morse.

"These lectures will be primarily on a theory of the calculus of variations in the large. Such a theory may be regarded as concerned with the most general boundary value problem involving extremals. It requires, I, a preliminary characterization of the solutions in the small, II, a topological study of the critical points of functions and, III, a combination of these results in the form of a general theory. The following topics will be treated.

"I. The Characterization of Solutions in the Small.

a. The Lagrange problem under general end conditions.

b. The associated linear boundary-value problems.

1. The algebraic properties of self-adjoint systems.

2. Separation, comparison, and oscillation theorems.

3. Sturmian conditions as focal point conditions.

4. Interrelations of conjugate points, focal points, and characteristic roots.

5. Periodic solutions.

c. The geometric invariance of characteristics of solutions. (Using tensor analysis.)

d. The semi-topological invariance of characteristics of solutions. (Using deformations.)

"II. The Critical Points of Functions.

a. The type numbers of critical sets.

1. The non-degenerate case.

2. The analytic case.

3. The general case.

4. The invariance of the type numbers.

b. Relations between the type numbers and the Betti numbers.

c. The completeness of the relations.

d. Applications to differential topology, potential theory, and dynamics.

"III. The Calculus of Variations in the Large.

a. One end point fixed. Ordinary connectivities.

b. Two end points fixed. Two point connectivities.

c. End points variable on two manifolds. Product connectivities.

d. Periodic extremals. Circular connectivities.

e. A method of topological continuation.

f. The Poincaré Theorem. Birkhoff's results in $n$ dimensions.

g. General formulations and topological analogies.

"The principal results to be treated have been presented in the literature only in part. The reader may refer to the recent papers by the author in the Transactions, to the Mathematische Annalen, volume 99, and to abstracts in the Proceedings of the National Academy of Sciences. Here will be found references to papers by Birkhoff, Brown, Bliss, Bolza, Richardson, Poincaré, Lusternik and Schnirelmann, Alexander, Lefschetz, Veblen, and others."

L. M. GRaves 